\title{
CURRENT ASPECTS OF USING ARTIFICIAL INTELLIGENCE IN DIGITAL GAMES AND COMPUTER GRAPHICS CONTENT CONTROL
}

\author{
Veljko Aleksić ${ }^{*}$, \\ Dionysios Politis ${ }^{2}$ \\ ${ }^{1}$ University of Kragujevac, \\ Faculty of Technical Sciences, \\ Čačak, Serbia \\ ${ }^{2}$ Aristotle University of Thessaloniki, \\ School of Sciences, \\ Thessaloniki, Greece
}

\begin{abstract}
:
The extraordinary size and influence of gaming industry on contemporary (digital) markets creates a vast pool of resources (material and abstract) that is constantly refilled and used in order to make modern digital games of all kinds and genres as attractive and performant as possible. A key component of almost every complex digital game that is developed today is its artificial intelligence module. Due to the variety of problems that can be and are solved by using artificial intelligence in digital games its functionality is practically essential.

The paper describes the application of artificial intelligence methods, its implementation in the content generation process, as well as modelling the gameplay. The game design directly conditions the complexity and performance of artificial intelligence module. It should be noted that the digital games that so far used mode advanced artificial intelligence techniques had variable success, which implies that this area of application still needs to be fully defined, and that the future technological/software progress will further determine the level of freedom that game designers will have in their creativity.
\end{abstract}

Keywords:

Digital Games, Computer Graphics, Artificial Intelligence.

\section{INTRODUCTION}

The field of artificial intelligence is rapidly evolving for the benefit of various industries and applications. The creation of interconnected and intraconnected machines, devices and software is enabled by using multidisciplinary and interdisciplinary approaches in the fields of mathematics, computer science, technology, psychology, art, linguistics, etc. When we carefully observe the fantastic world of digital games as one of the highest software abstractions or entities, it is natural that the question of managing and controlling this incredibly complex digital environment must come in order. Interestingly, even though they are incredibly complex, digital games bear probably the least risk to human life and wellbeing in real-world application of all IT industry fields, if you exclude the possibility of one developing digital game addiction, of course.
Correspondence:

Veljko Aleksić

e-mail:

veljko.aleksic@ftn.kg.ac.rs 
The importance and potential of digital games industry have been recognised for the last half century, as the IT gradually entered everyday human lives and gaming became primary leisure human activity today.

Digital games are important application of artificial intelligence today, and presents an excellent platform for implementing adaptive intelligent agents. As a base, machine learning can naturally make digital games more interesting and reduce the production cost.

The rest of the paper is organized as follows. The Section 2 focuses on the typical applications of artificial intelligence in digital games. Subsequentially, the novel algorithmic approaches that are used in contemporary digital gameplay design are described in brief following the application of artificial intelligence in procedural content creation and player modelling. Section 3 gives concluding remarks on the topic.

\section{THE APPLICATION OF ARTIFICIAL INTELLIGENCE IN DIGITAL GAMES}

The historical development of digital games greatly correlates with the development of artificial intelligence and neural networks. For a long time, artificial intelligence managed gameplay agents combined with learning components was the primary approach in this symbiosis [1]. Early research mainly focused on more traditional game concepts with simple rules but great complexity in solving, such as Chess (e.g., IBM Deep Blue [2]). During the late 20th century (e.g., 80's), another alternate use of artificial intelligence emerged - procedural content creation. One of the first famous examples of successful implementation in the field of home PC gaming market was the Elite, a space simulation game that used artificial intelligence for creating vast simulated universes with their unique star systems and planets without the need for manual static design which greatly reduced the memory requirements [3]. This was extremely important at the time when home computers were scarce and very limited in processing power, memory and storage space. Even though computers are nowadays digital data powerhouses, previous application modality is still being used extensively. Existing procedural content generation methods, such as search-based, solver-based, rule-based and grammarbased methods have been applied to various content types such as levels, maps, character models, and textures [4]. For the last two decades, artificial intelligence started being used in analysing gameplay characteristics and modelling player profiles.
As the global gaming market exploded thanks to the internet (among other factors) it became very important for game designers to appeal very diverse audience, in age, interests, culture, language, financial background, digital literacy, religion, etc. Nowadays, practically every successful commercial game continuously collects gameplay data and analyses it with the support of artificial intelligence agents [5][6], and even consumer electronics manufacturers started providing artificial intelligence-based metrics to (semi) professional players, e.g., HP OMEN Command Center (Fig. 1).

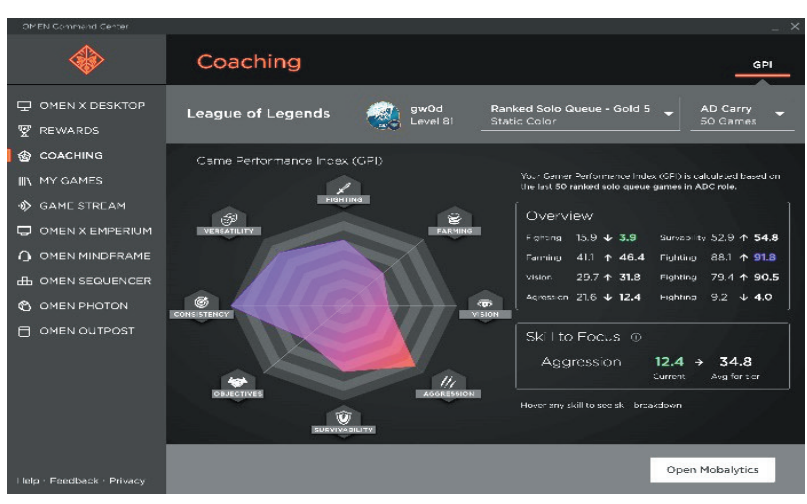

Figure 1 - AI coach in HP OMEN Command Center

\subsection{KEY GAMEPLAY AI ALGORITHMS}

When we talk about modern digital games, finite state machines, behavior trees, and utility-based artificial intelligence are authorship methods that traditionally dominated the control of non-player characters (NPCs) [7]. Finite state machine (FSM) and the hierarchical finite state machine (HFSM) variant were mostly used in NPC control and decision-making processes a decade ago.

Contrary to supervised learning where agent is provided with the action as a response to various states (e.g., human labelled data was provided for the algorithm to learn patterns), Reinforcement Learning agent interacts with the environment and usually learns to perform through rewards. Maybe the biggest challenge in successful applying reinforcement learning is balancing short- and long-run high rewards, i.e., exploit vs explore [8]. Still, random trial and error is a key component and the method by which agent must begin leaning to receive a reward. Unlike other machine learning techniques, reinforcement learning is not limited to (human) labelled data. Still, it should be noted that the examples of successful implementation of reinforcement 
learning in commercial games are extremely scarce (e.g., Black \& White) as It demands extensive data manipulation and customization.

By combining reinforcement learning and deep neural networks researchers created algorithm that can learn directly from sensory streams - Deep Reinforcement Learning [9]. Images, videos and sounds can all present a valid high-dimensional input that the algorithm can use without pre-processing. Deep reinforcement learning algorithm is often used in modern games (e.g., StarCraft, MOBA, Dota2, Minecraft) [10]. Even though the algorithm is one of the most promising, some challenges for its wider implementation in digital games such as balancing between exploration and exploitation, low sample efficiency, problems with generalization, multi-agent learning, etc., still need to be addressed.

Deep Learning is a broad term but when we talk about digital games the idea is to progressively learn high-level features through layers of nonlinear processing. Efficient fast GPUs and affordable video memory enabled DL methods to surpass image and speech recognition applications towards even more complex cases, such as automatically learning player models that are used for fast artificial playtesting of new levels during game development [11] (Fig. 2). Recent advances in deep learning have been additionally accelerated because of the increased interest by a variety of different companies such as Facebook, Google/Alphabet, Microsoft and Amazon, which heavily invest in its development [12].

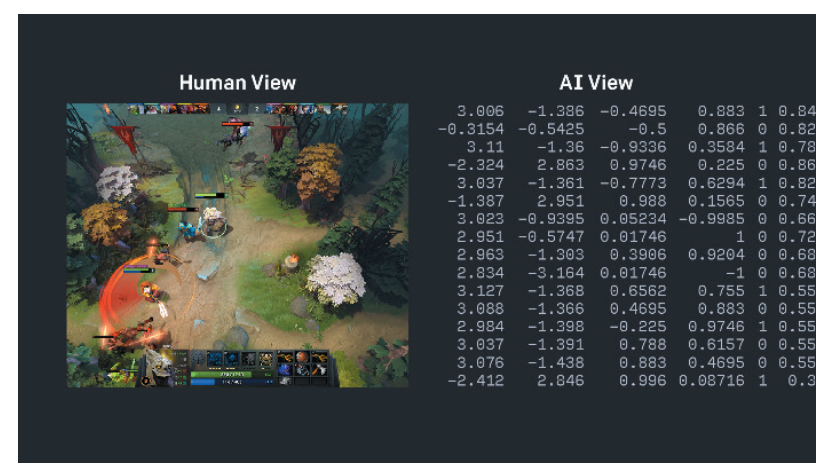

Figure 2 - OpenAI Five playing Dota2

Contrary to the traditional alpha/beta game development sequence, Monte Carlo Tree Search algorithm uses controlled explore \& exploit of previously obtained knowledge and often fully randomized playouts.
This algorithm can be applied to large trees but cannot guarantee optimal solution. Win/Loose information is propagated up to the tree root and every node holds an estimate of the win ratio so the search can be directed. One of the most successful implementations of the Monte Carlo Tree Search algorithm is controlling opponent human-like behaviour in fighting games [13][14][15].

When traditional methods of optimization stalls or cannot be applied because of the lack of numeric objective values Evolutionary Algorithms comes to light. The idea is to do parallel search by concurrently improving means of populations of various candidate solutions. This flexibility enabled evolutionary algorithm often outperforming deep learning machines playing digital games. As it currently stands, evolutionary algorithm will be in the focus of great number of researches for its further application in digital game design and production in near future.

\subsection{PROCEDURAL CONTENT CREATION}

Using artificial intelligence in digital game procedural content creation increases its replayability by offering players new experience every time they play.

For example, Civilization series (Fig. 3), World of Warcraft: Shadowlands, Microsoft Flight Simulator, Minecraft, etc., all use procedural content generation methods as part of their core gameplay engine which enable player exploring practically unlimited varieties of digital gameplay environments and the creation of extremely large game worlds even on devices with relatively modest memory capacity, such as low-spec computers and smartphones.

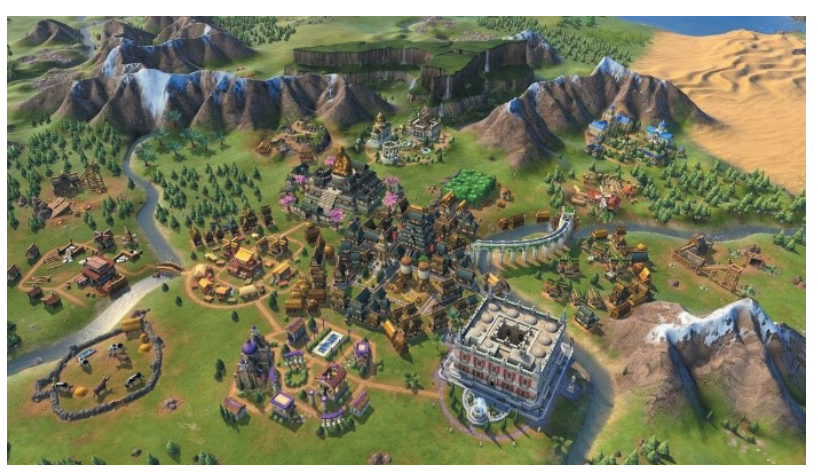

Figure 3 - Civilization VI uses procedural content creation method for world map customization 
One interesting and very applicable filed of using procedural content creation approach is in training deep reinforcement learning artificial intelligence agents. As a matter a fact, using deep reinforcement learning in playing digital games has one crucial flaw - if the environment that agent trained on is not exactly the same as in the actual game - the method overfits. By using procedural content creation in their training, agent performance became significantly more general [16].

\subsection{GAME ANALYTICS AND PLAYER MODELLING}

Game analytics is currently the main use case of artificial intelligence in digital games industry. In addition to previously mentioned learning to play and content creation, game analytics is crucial in collecting data about the player while in the game and then ad hoc updating the game so for instance the difficulty level could be automatically adjusted accordingly or user interface could be streamlined [17].

Another important application of artificial intelligence in adaptive gameplay paradigm is player modelling. Modelling can be executed via supervised (e.g., training a neural network on recorded human gameplay) or unsupervised learning. The combination of procedural content creation and player modelling allows the algorithms to automatically generate unique content in accordance to player desired experience [18]. This approach is called Experience-Driven Procedural Content Generation.

A decade ago, focus of many researchers was the human-like behaviour of NPCs, and with the new interest in human-AI collaboration it is likely that it will be in the spotlight again.

Debugging and playtesting during game design and production is another field of artificial intelligence application that shown some encouraging results. Balancing game mechanics is crucial to the gameplay quality and the success of game in general, and is still underresearched area that may be exploited.

\section{CONCLUSION}

As digital game development companies and studios are more and more collaborating with academic research institutions, latest innovations in artificial intelligence will surely find their commercial application quickly. Procedural content creation and deep reinforcement learning are already a part of some of the biggest gaming franchises, so their further development and implementation is secured. Near future will probably bring into play a combination of some machine learning techniques and procedural content creation [19], such as Generative and Adversarial Networks. This will enable players further personalisation of their game avatars in a level so far unprecedented and realistic.

Future applications of artificial intelligence in digital games will probably lead to better collaboration between various agents, better automated playtesting and natural language processing to the extent that at some point there will be free-form direct communication between players and NPCs.

In conclusion, the desire to apply more artificial intelligence algorithms to complex digital games is clear, but the real-life implementation has many obstacles. Starting with having very little control over the deep learning methods actual performance to hardware limitations when experimenting with parallel computation. It is obvious that only a limited portion of academic research in the field of artificial intelligence is actually applicable when we observe digital game industry.

\section{REFERENCES}

[1] F. Dignum, J. Westra, W. A. van Doesburg, and M. Harbers, "Games and Agents: Designing Intelligent Gameplay," International Journal of Computer Games Technology, vol. 2009, pp. 1-18, 2009.

[2] M. Campbell, A. J. Hoane, and F. Hsu, "Deep Blue," Artificial Intelligence, vol. 134, no. 1-2, pp. 57-83, Jan. 2002.

[3] S. Risi and M. Preuss, "From Chess and Atari to StarCraft and Beyond: How Game AI is Driving the World of AI," KI - Künstliche Intelligenz, vol. 34, no. 1, pp. 7-17, Feb. 2020.

[4] J. Liu, S. Snodgrass, A. Khalifa, S. Risi, G. N. Yannakakis, and J. Togelius, "Deep learning for procedural content generation," Neural Computing and Applications, vol. 33, no. 1, pp. 19-37, Oct. 2020.

[5] B. Cowley, I. Kosunen, P. Lankoski, J. M. Kivikangas, S. Järvelä, I. Ekman, J. Kemppainen, and N. Ravaja, "Experience Assessment and Design in the Analysis of Gameplay," Simulation \& Gaming, vol. 45, no. 1, pp. 41-69, Dec. 2013.

[6] R. Marczak, G. Schott, and P. Hanna, "Postprocessing Gameplay Metrics for Gameplay Performance Segmentation Based on Audiovisual Analysis," IEEE Transactions on Computational Intelligence and AI in Games, vol. 7, no. 3, pp. 279-291, Sep. 2015. 
[7] X. Zhu, "Behavior tree design of intelligent behavior of non-player character (NPC) based on Unity3D," Journal of Intelligent \& Fuzzy Systems, vol. 37, no. 5, pp. 6071-6079, Nov. 2019.

[8] M. Jaderberg, W. M. Czarnecki, I. Dunning, L. Marris, G. Lever, A. G. Castañeda, C. Beattie, N. C. Rabinowitz, A. S. Morcos, A. Ruderman, N. Sonnerat, T. Green, L. Deason, J. Z. Leibo, D. Silver, D. Hassabis, K. Kavukcuoglu, and T. Graepel, "Human-level performance in 3D multiplayer games with population-based reinforcement learning," Science, vol. 364, no. 6443, pp. 859-865, May 2019.

[9] R. R. Torrado, P. Bontrager, J. Togelius, J. Liu, and D. Perez-Liebana, "Deep Reinforcement Learning for General Video Game AI," 2018 IEEE Conference on Computational Intelligence and Games (CIG), Aug. 2018.

[10] I. Oh, S. Rho, S. Moon, S. Son, H. Lee, and J. Chung, "Creating Pro-Level AI for a Real-Time Fighting Game Using Deep Reinforcement Learning," IEEE Transactions on Games, pp. 1-1, 2021.

[11] S. F. Gudmundsson, P. Eisen, E. Poromaa, A. Nodet, S. Purmonen, B. Kozakowski, R. Meurling, and L. Cao, "Human-Like Playtesting with Deep Learning," 2018 IEEE Conference on Computational Intelligence and Games (CIG), Aug. 2018.

[12] N. Justesen, P. Bontrager, J. Togelius, and S. Risi, "Deep Learning for Video Game Playing," IEEE Transactions on Games, vol. 12, no. 1, pp. 1-20, Mar. 2020.

[13] R. Ishii, S. Ito, M. Ishihara, T. Harada, and R. Thawonmas, "Monte-Carlo Tree Search Implementation of Fighting Game AIs Having Personas," 2018 IEEE Conference on Computational Intelligence and Games (CIG), Aug. 2018.

[14] M. Ishihara, S. Ito, R. Ishii, T. Harada, and R. Thawonmas, "Monte-Carlo Tree Search for Implementation of Dynamic Difficulty Adjustment Fighting Game AIs Having Believable Behaviors," 2018 IEEE Conference on Computational Intelligence and Games (CIG), Aug. 2018.

[15] I. P. Pinto and L. R. Coutinho, "Hierarchical Reinforcement Learning With Monte Carlo Tree Search in Computer Fighting Game," IEEE Transactions on Games, vol. 11, no. 3, pp. 290-295, Sep. 2019.

[16] C. E. López, J. Cunningham, O. Ashour, and C. S. Tucker, "Deep Reinforcement Learning for Procedural Content Generation of 3D Virtual Environments," Journal of Computing and Information Science in Engineering, vol. 20, no. 5, Jun. 2020.

[17] A. Drachen and S. Connor, "Game Analytics for Games User Research," Oxford Scholarship Online, Mar. 2018.
[18] G. N. Yannakakis and J. Togelius, "ExperienceDriven Procedural Content Generation," IEEE Transactions on Affective Computing, vol. 2, no. 3, pp. 147-161, Jul. 2011.

[19] S. Risi and J. Togelius, "Increasing generality in machine learning through procedural content generation," Nature Machine Intelligence, vol. 2, no. 8, pp. 428-436, Aug. 2020. 